\title{
Some Features of Liquid Crystalline Oxadiazoles
}

\author{
Amelia Carolina Sparavigna ${ }^{1}$ \\ ${ }^{1}$ Department of Applied Science and Technology, Politecnico di Torino, Corso Duca degli Abruzzi 24, Torino, Italy
}

\begin{abstract}
We propose a survey of some features of those oxadiazole compounds which are mesogenic, that is, which are displaying liquid crystalline properties induced by their rod- or disk-like molecules. We will see that they can have bend-shaped molecules too, creating biaxial phases, and other interesting peculiarities in the nematic and smectic phases. With large electric dipoles and luminescent properties, these materials are also very appealing for technological applications.
\end{abstract}

\section{Introduction}

Certain oxadiazoles are mesogenic compounds possessing the typical liquid crystalline nematic and smectic mesophases. Even the elusive biaxial nematic phase is displayed: this peculiar phase, theoretically proposed in 1970 by Freiser, can be observed in the mesophase of oxadiazoles with boomerang-shaped molecules [1-3]. Therefore, oxadiazoles are rather interesting for both experimental and theoretical researches. However, these compounds are relevant for applications too, in particular in the electroluminescent devices where they are the emissive materials [4].

Before starting a short survey of researches and results obtained with these compounds, let us discuss the basic structure of the oxadiazole molecule, because it is mainly the oxadiazole group that gives the shape to the final molecule in which it is inserted.

As generally told, the liquid crystal compounds are mesogenic due to their molecular rod-like or disk-like shape. However, the role of the molecular shape in the features of mesophases is more complex. If the rodlike structure of the molecule is bent for instance, the material can produce a spontaneous symmetry breakdown, as in the case of the banana-shaped molecules studied by Niori et al.[5]. This experimental discovery by the Tokyo Technology group in 1996, of a ferroelectric behavior in a mesophase of a compound having achiral bend-core (banana) molecules, represented a revolution for the liquid crystals community. It is then not surprising the persistent interest for compounds where the shape of molecules is not simply rod-like or disk-like: oxadiazoles are among them.

The oxadiazole is an aromatic heterocyclic molecule, which structure is shown in the Figure 1. It has carbon, hydrogen, nitrogen and oxygen. In fact, it contains two nitrogen atoms (for instance, oxazole has one nitrogen atom). There are four possibilities to arrange nitrogen and oxygen, as the figure shows. Moreover, we have the possibility to substitute or di-substitute the hydrogen in the other two positions. An example is the 1,2,5-oxadiazole-3,4-diamine compound in the same figure. We can easily imagine how some longer threads attached to the oxadiazole, substituting diamine groups, can create a possible mesogenic compound with a bend-core shape. For this reason, the oxadiazole was used to build the boomerang-shaped molecules.

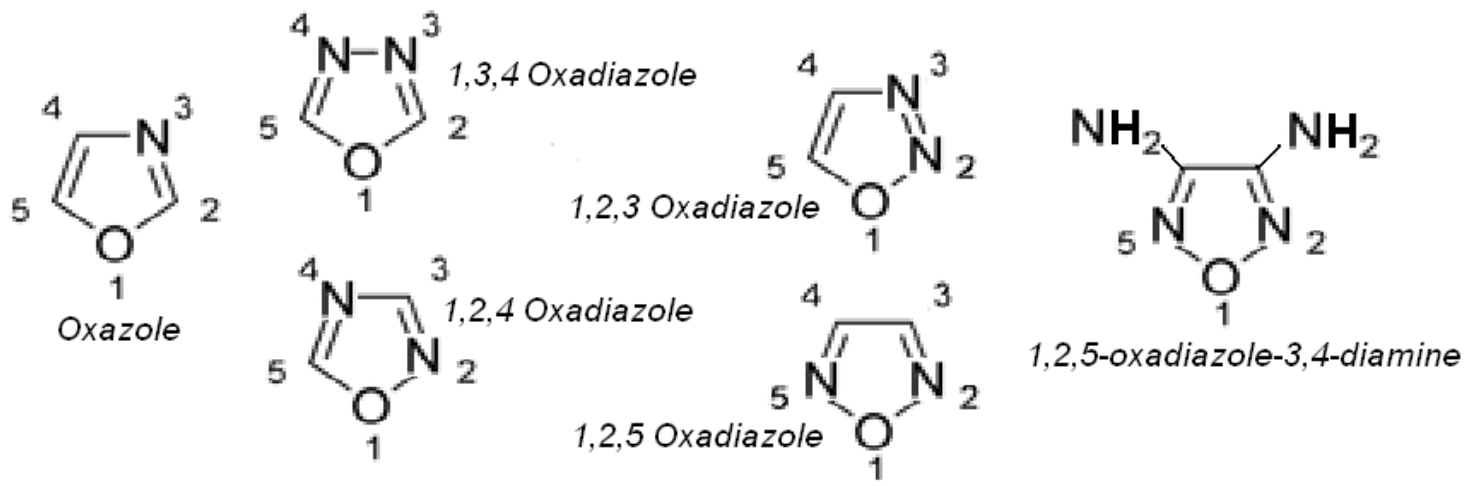

Figure 1 The four oxadiazoles in the middle, the oxazole on the left and an example of a substance with the 1,2,5oxadiazole on the right.

\section{Amelia Carolina Sparavigna (Correspondence)}

d002040@polito.it 


\section{Symmetric compounds with 1,3,4-oxadiazoles with biaxial features.}

References [1,2] show the boomerang molecules obtained with the 1,3,4-oxadiazole. These molecules have a biaxial molecular arrangement in the smectic A phase. But they have a biaxial nematic too. This is a mesophase different from the uniaxial nematic phase, where three distinct optical axes are present. The uniaxial phase has a single preferred axis, around which the system is rotationally symmetric $D_{\infty \mathrm{ch}}$. The symmetry group of a biaxial nematic is $\mathrm{D}_{2 \mathrm{~h}}$. This group can be represented by the geometry of a rectangular right parallelepiped. And in fact, a route to biaxiality in nematics is the development of molecules, which have the shape of parallelepipeds.
Numerical simulations [6] of transitions, from the ordinary nematic to the biaxial one, give the evolution of schlieren textures as they can be observed with the polarized light microscope. It results that the problem is more complex than what is commonly suggested, that it is the charge of disclinations which distinguishes the biaxial form from the uniaxial phase. To see beautiful images obtained with polarized microscope of such transition, let us look at Reference 7, where mesophases of 1,4"-p-terphenyl-bis-2,3,4-tridodecyloxy-benzal-imine are investigated. This is a calamitic compound with a biaxial nematic phase. An example of how the texture transition appears, when the nematic passes from uniaxial to biaxial symmetry, inspired from images in [7], is shown in the Figure 2.
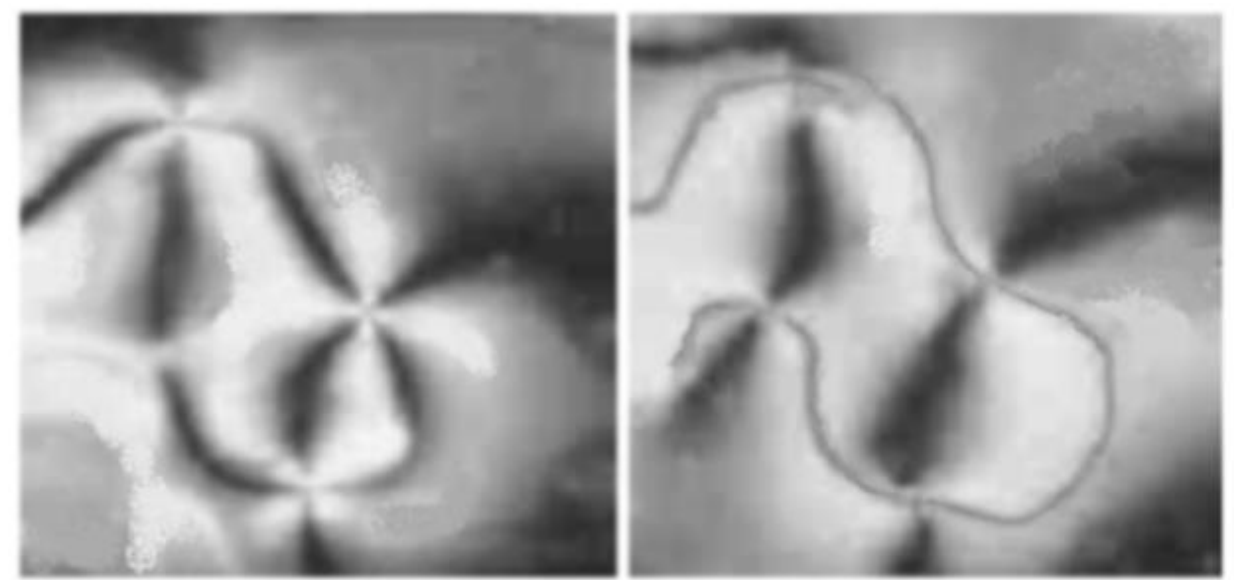

Figure 2 The image illustrates the transition from uniaxial (on the left) to biaxial (on the right) nematic phases as shown by the schlieren texture observed by means of the polarized light microscopy.

In spite of the fact that a biaxial nematic prefers to have a schlieren texture with disclinations of strength $1 / 2$, unambiguous results are given by $\mathrm{H}^{2}$-NMR investigations [1]. The resonance demonstrates that ODBP-Ph- $\mathrm{C}_{7}$ and ODBP-Ph- $\mathrm{C}_{12}$ have a truly biaxial nematic (see Figure 3 for the molecular structure of such compounds). The researchers defined these molecules as boomerangs, because the angle at the core of the molecule, which is the angle determined by the oxadiazole group, is approximately of $140^{\circ}$, greater than the typical value of $120^{\circ}$ of banana mesogenic molecules. There are of course biaxial-shaped calamitic materials, but ODBP boomerangs have a large electric dipole $(\approx 4 \mathrm{D})$, which adds the possibility of strong intermolecular association.

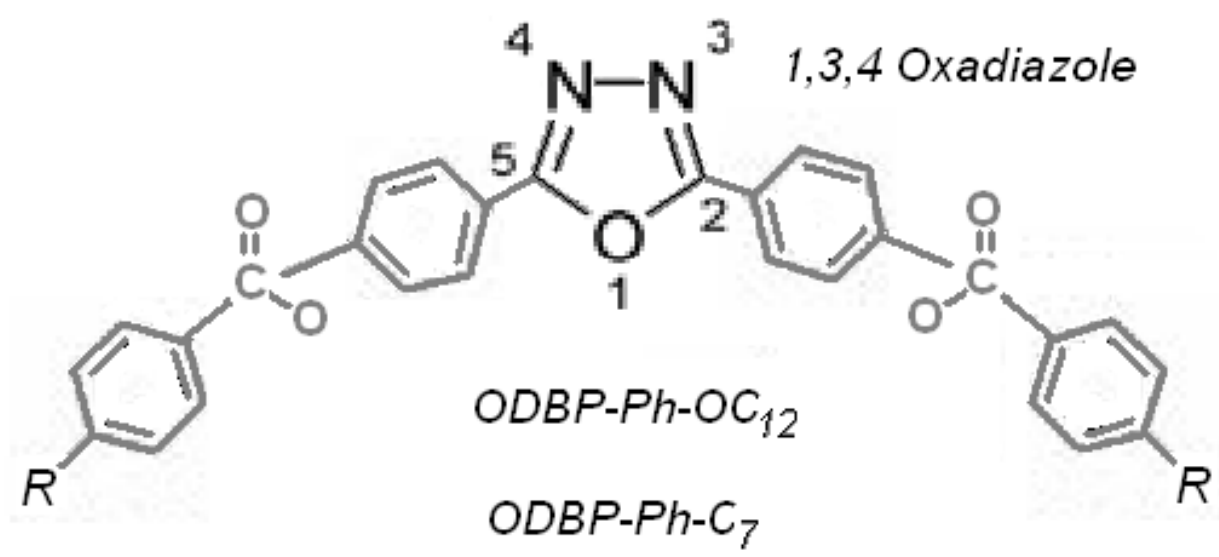

Figure 3 Boomerang molecules. $R$ is the different fragment $O C_{12}$ or $C_{7}$. 


\section{The 2,5-disubstituted-1,3,4-oxadiazoles}

Many compounds with 1,3,4 oxadiazoles have been prepared to study their luminescence property [4]. The compounds are generally chemically stable, sometimes with good electron-transport properties. The study on these materials allows the conclusions that the presence of mesophases is affected by both electronic and steric factors within terminal substituents [4]. Smectic and nematic, enantiotropic and monotropic phases were observed with polarized light microscope. Let us remember that an enantiotropic phase can be observed both heating and cooling the material, whereas a monotropic phase can be entered only during the heating or the cooling, not both.

In Reference 4, it is reported how a large molecular dipole can be obtained inserting a fluorine-substituent in oxadiazoles with 2,5-diphenyl-1,2,3-oxadiazole (electron-deficient) and p-alkoxyphenyl (electronrich) moieties. F atom, having a strong tendency to gain an electron, perturbs the charge distribution producing the dipole. The insertion of $\mathrm{F}$ atom is then stabilizing, whereas the insertion of electron-donor substituents destabilizes the mesophases. Liquid crystalline oxadiazole compounds with halogens (both 1,3,4-oxadiazoles and 1,2,4-oxadiazoles, see Figure 4) were investigated also in the Refs.[8-10].

The importance of oxadiazole compounds is due to the simultaneous presence of a bend-shape and of an electric dipole; this fact is very attractive in the framework of researches on materials with achiral molecules, which could display ferroelectric states. The fact that the dipolar nature of the oxadiazole core is influencing the mesomorphism was shown by molecular simulations too [11].
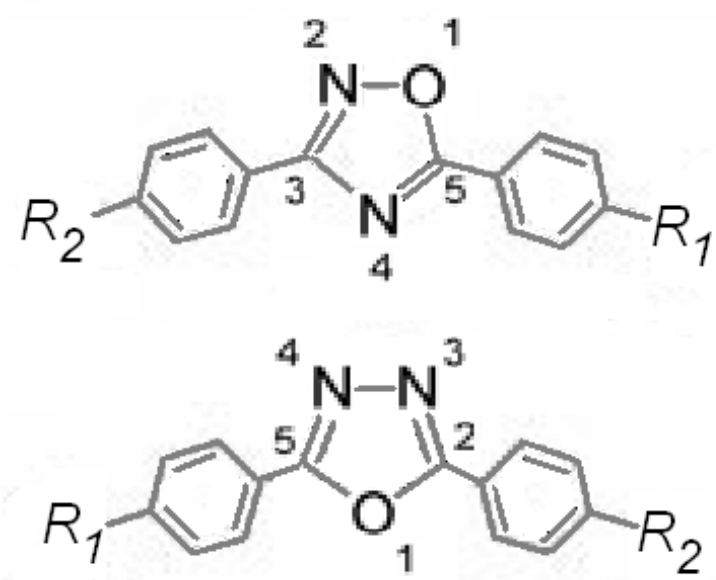

Figure 4 ODBPs structures with 1,3,4 and 1,2,4 oxadiazoles.

$R_{1}$ and $R_{2}$ are substituents with can be halogen atoms.

\section{The 3,5-disubstituted-1,2,4 oxadiazoles with bend-shape}

The Liquid Crystal Group of the Organic Intermediates and Dyes Institute in Moscow was the first group to study 3,5-disubstituted 1,2,4-oxadiazoles as fragments of liquid crystalline compounds [8-10]. Before their studies, these compounds were used only for pharmaceutical purposes. In Ref.12, several asymmetric heterocyclic structures are proposed, leading to molecules with bend-shape and mesogenic properties (Figure 5). The 1,2,4-oxadiazole compounds display distinct aspects such as an asymmetrical distribution of electronic density, giving a dipole moment of $\approx 1.5 \mathrm{D}$. A distortion of the linearity of the molecule due to the bonding angles is observed and then the authors named the compounds as bananashaped 1,2,4-oxadiazoles.

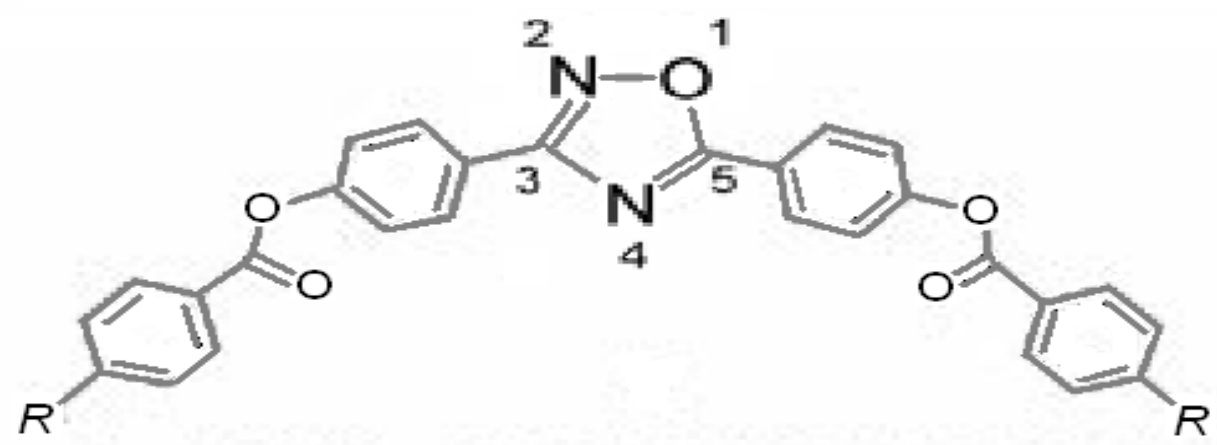

Figure 5 A banana-shaped compound from Ref.12. 


\section{Asymmetric oxadiazoles}

The correlation between the existence of mesophases and the chemical structure of molecules is always important for the research in liquid crystals. When a family of asymmetric structures is under investigation, the variety of results is strongly enhanced. This is true for mesomorphic oxadiazole compounds too. Then, asymmetric oxadiazoles have been developed [8], because of their molecular structure with heterocycles and several possible substituents, providing a variety of smectic and nematic mesophases. The examples we can find in the references previously reported, show that not only the chemical structure of the substituents, but also their position with respect to the oxadiazole ring is relevant for the mesophases.

The optical microscope investigations show very interesting behavior of the smectic and the nematic phase of some of these oxadiazole compounds [13]. In particular the smectic phases have a toric texture that changes in a spherulitic nematic. Moreover, it is possible to observe a remarkable behavior in the nematic phase of 3,5-disubstituted-1,2,4-oxadiazoles, a texture transition driven by the temperature. We consider a "texture transition" as a change in the nematic texture observed by means of the polarized light microscope. The low temperature nematic has a texture with spherulitic domains. Figure 6 shows the transition between the smectic and the spherulitic nematic of compound C of Ref.13 (here simply called ODBP). The figure shows the smectic (a) and the lowtemperature (b) and the high-temperature (c) nematic phases.

Texture transitions have been previously observed inside the nematic phase of some mesomorphic thermotropic materials belonging to the families of the alkyloxybenzoic and cyclohexane acids [14-16]. In some cases, peaks in DSC scanning and dielectric spectroscopy investigations accompany the evidence of the texture transition observed by polarized light microscopy. [17]. The Figure 6 in its upper part shows the smectic (a) and the two nematic phases (b) and (c) of the alkyloxybenzoic acid HOBA.

Alkyloxybenzoic and cyclohexane acids have mesophases because the mesogenic units are hydrogen-bonded dimers. The more common explanation for the presence of a texture transition in the nematic range is based on the existence of cybotactic clusters of such dimers, favoring a local smectic order in the nematic range [18]. Of course, the texture transition inside the nematic phase of oxadiazole compounds could be due to surface effects, but we suggest a possible existence of cybotactic clusters in these materials too. In Reference 2, the possibility of cybotactic cluster is also reported. Probably, this is a biaxial feature; however this fact needs to be studied by further investigations.
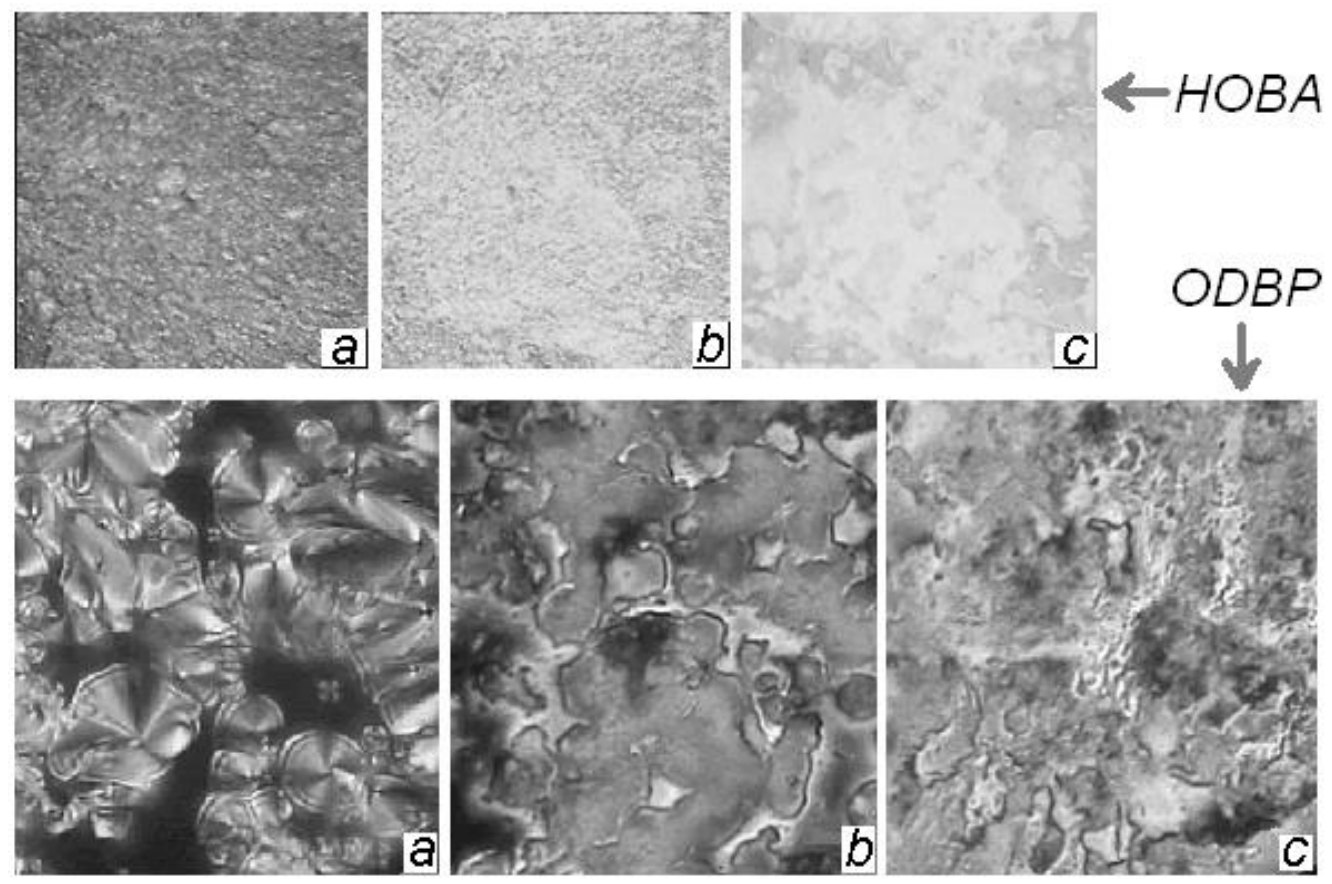

Figure 5 Smectic and nematic phases of HOBA [16] and ODBP (compound C in [13]). The smectic phase (a) turns in a nematic with smectic-like texture $(b)$. This nematic transforms into an ordinary nematic $(c)$ when subjected to further increase of temperature. 
Hydrogen-bonded oxadiazoles have been also prepared and studied [19], to understand the role of an increased flexibility of molecules. One of the parameters of materials with bend-core molecules, relevant for mesomorphism, is the bend angle. If this angle is too small, the mesomorphism is usually suppressed. For large angles, we have the boomerang oxadiazoles, which display the biaxial phase, where molecules have 140 degrees for the bend angle. If the angle is greater, the behavior of the system is expected to be that of a calamitic material. The suggestion in [19] is therefore to use flexible linked dimers, to tune the value of the bend angle and check if and when a biaxial or a calamitic behavior is observed.

Asymmetric molecules containing metals and 1,3,4oxadiazole and 1,3,4-thiadiazole heterocycles have been proposed too [20]. These materials are named as "metallomesogens" when are exhibiting thermotropic mesophases and are classified according to the bond formed between the metal and the organic molecule. The interest in synthesizing of metal-containing liquid crystals is due to some advantages in combining the properties of liquid crystal systems with those of transition metals, for applications in optical devices.

\section{Oxadiazoles for electroluminescence}

Organic compounds mostly behave like insulators. But organic compounds with proper structure and orientation of molecules can transport electric current upon certain conditions, competing then with the conventional materials used in electronics. Chains of polythiophenylenes for instance, have a charge transport comparable to poor metallic conductors.

In electronics, organic compounds can operate as rectifiers if they have a donor electron-rich part and an acceptor electron-poor part, linked by an insulating bridge. The lowest unoccupied molecular orbital (LUMO) of the acceptor and the highest occupied molecular orbital (HOMO) of the donor are used for transport of electrons. Some organic compounds with proper HOMO and LUMO orbitals can emit light when an electric current is passing through them. This is the electroluminescence. It is based on an electron transfer from LUMO and HOMO orbitals. The molecule in an excited state comes back to its ground state by an electron transfer from LUMO to HOMO orbital, accompanied by irradiation of energy difference in the form of light.

Materials that can simultaneously act as liquid crystals, charge transport agents and emitters are of interest for potential applications in organic light emitting diodes. In 1990, it was found that a compound with 1,3,4oxadiazole is an excellent electron transport material in an organic electroluminescent diode [21]. After this report, the research to use various oxadiazole molecules to obtain high electroluminescence performances strongly increased. The researches on polymer light-emitting diodes showed that the oxadiazole moieties possess a high potentiality for transport [22,23].

Discotic oxadiazoles, with columnar mesophases, were proposed too, for applications in organic electronics in Ref.24. Recently [25], star-shaped molecules consisting of a 1,3,4-oxadiazole core were synthesized. These molecules have columnar mesophases over a wide temperature range, where the mesophases exhibiting strong blue fluorescence. It is interesting to note that the researchers found that on cooling, one compound transforms into a transparent glass at room temperature, where the mesophase texture was retained. This glassy film is exhibiting blue luminescence, with an absolute quantum yield of $26 \%$. The team observed that the length of the alkyl substituents inserted in the molecules has a significant effect on the absorption and fluorescence properties of the materials, due to the role of the alkyl substituents in controlling the nature of the molecular packing.

The introduction of oxadiazole moieties to polymer main chains and to mesogens in liquid crystalline compounds is then used to tune the electroluminescence efficiency and the transport properties. Electroluminescent polymers were also prepared, in which thiophene and oxadiazole moieties are connected alternately to form fully conjugated polymers. Likewise, liquid crystalline compounds containing oxadiazole moieties were reported to exhibit a high electron transport capability and a blue electroluminescence emission $[22,26]$.

Another recent research is proposing new oxadiazole compounds containing silane, which have high morphological stability [27]. The researchers found that the disruption of the $\pi$-aromatic conjugation by introduction of $\mathrm{Si}$ atoms leads to a large band gap and high triplet energy. They identified among their new compounds the best host for FIrpic, to have a phosphorescent organic light emitting diode (PHOLED). Let us remember that in OLED, two modes of light emission have to be considered: fluorescence and phosphorescence. OLEDs using fluorescent materials are limited by the internal quantum efficiency, whereas in phosphorescent materials the internal quantum efficiency can reach up to $100 \%$. Classical examples of phosphorescent emitting materials have green and red emitters. In order to attain blue emission, FIrpic, a prototypical blue-green emitter is used, where the heteroleptic iridium complex contains two fluoro-substituted phenylpyridine ligands and an anionic 2-picolinic acid [28].

\section{Enantioselective segregation and drugs}

To conclude, let us see another reason to investigate oxadiazoles. Studying the oxadiazoles which display 
the biaxial nematic phase [29], Görtz and Goodby found that the achiral biaxial nematic phase can segregate into chiral domains of opposite handedness. In fact then, they observed a nematic phase which exhibited the properties of a conglomerate. The enantioselective segregation has been obtained in the smectic and nematics phases of other banana oxadiazoles, without introducing any chiral species, by a Japanese group [30]. This sort of segregation is rather important for drugs, because the stereoisomers of chiral drugs often exhibit pronounced differences in their properties, so pronounced that it is necessary to study each stereoisomer separately [31]. This is the reason why the pharmaceutical research has converged to use single enantiomers as substitutes for their racemates. And then, new developments in medicine could start from liquid crystals laboratories, with further investigations on boomerang or bananaoxadiazoles.

In Section 4 we have already observed the fact that 1,2,4-oxadiazoles are significant in terms of their pharmacological properties. For instance, the 1,2,4oxadiazole systems is used in the Oxolamine which is a cough suppressant. Oxadiazoles are antibacterial, antimalarial, anti-inflammatory, antifungal and anticonvulsant (see for instance, [32] and [33]). Recently [34], some 2,5-substituted diphenyl-1,3,4oxadiazoles have been studies for their biological activities of the $-\mathrm{N}=\mathrm{C}-\mathrm{O}-$ grouping. According to the authors, the derivatives of 1,3,4-oxadiazole are versatile hydrophobic molecules with biological activities, with lesser or limited amount of toxicities. And therefore, the researchers consider them as most promising compounds in antidepressant, anticonvulsant and antianxiety activity with no neurotoxicity when compared with standard.

Let us conclude that, even if oxadiazole compounds have been explored largely in the past, several recent publications on their role in electronics and in the development of new drugs tell that new oxadiazoles are constantly produced for research purposes and investigated for future applications.

\section{References}

[1] L.A. Masden, T.J. Dingemans, M.Nakata and E.T. Samulski, Thermotropic biaxial nematic liquid crystals, Phys. Rev. Lett. 92, 145505 (4 pages), 2004.

[2] T.J. Dingemans and E.T. Samulski, Non-linear boomerangshaped liquid crystals derived from 2,5-bis(p-hydroxyphenyl)-1,3,4oxadiazole, Liquid Crystals 27, 131-136, 2000.

[3] M.J. Freiser, Ordered states of a nematic liquid, Phys. Rev. Lett. 24, 1041-1'43, 1970.

[4] Jie Han, S. Sin-Yin Chui, Chi-Ming Che, Thermotropic liquid crystals based on extended 2,5-disubstituted-1,3,4-oxadiazoles: structure-property relationships, variable-temperature powder X-ray diffraction, and small-angle X-ray scattering studies, Chem. Asian J. 1,814-825, 2006.

[5] T. Niori, F. Sekine, J. Watanabe, T. Furukawa and H. Takezoe, Distinct ferroelectric smectic liquid crystals consisting of banana shaped achiral molecules, J. Mater. Chem., 6, 1231-1233, 1996.

[6] C. Chiccoli, I. Feruli, O. D. Lavrentovich, P. Pasini, S.V. Shiyanovskii and C. Zannoni, Topological defects in schlieren textures of biaxial and uniaxial nematics, Phys. Rev. E 66, 030701 (4 pages), 2002.

[7] S. Chandrasekhar, Geetha G. Nair, D. S. Shankar Rao, S. Krishna Prasad, K. Praefcke and D. Blunk, A thermotropic biaxial nematic liquid crystal, Current Science, 75(10), 1998, available at http://www.iisc.ernet.in/currsci/nov251998/articles21.htm

[8] L.A. Karamysheva, S.I. Torgova, I.F. Agafonova and N.M. Shtikov, Dependence of mesomorphic properties of 3,5-disubstituted 1,2,4-oxadiazoles on geometric and electronic factors, Mol. Cryst. Liq. Cryst. 160, 217-225, 1995.

[9] O. Francescangeli, L.A. Karamysheva, S.I. Torgova, A. Sparavigna and A. Strigazzi, X-ray investigation of new isomeric oxadiazoles, Proceedings of SPIE 3319, 139-144, 1998.

[10] L.A. Karamysheva, I.F. Agafonova and S.I. Torgova, New heterocyclic analogs of $\mathrm{BCH}-$ and $\mathrm{CBC}$-compounds, Mol. Cryst. Liq. Cryst. 332, 407-414, 1999.

[11] J. Peláez and M.R. Wilson, Atomistic simulations of a thermotropic biaxial liquid crystal, Phys. Rev. Lett. 97, 267801 (4 pages), 2006.

[12] S.I. Torgova, T.A. Geivandova, O. Francescangeli and A Strigazzi, Pramana, Banana-shaped 1,2,4 oxadiazoles, 61(2), 239248, 2003 .

[13] A. Sparavigna, A. Mello and B. Montrucchio, Fan-shaped, toric and spherulitic textures of mesomorphic oxadiazoles, Phase Trans. 80, 987-998, 2007.

[14] A. Sparavigna, A. Mello and B. Montrucchio, Texture transitions in the liquid crystalline alkyloxybenzoic acid 6OBAC, Phase Trans. 79, 293-303, 2006; A. Sparavigna, A. Mello and B. Montrucchio, Texture transitions in binary mixtures of 6OBAC with compounds of its homologous series, Phase Trans. 80, 191-201, 2007.

[15] M. Petrov, A. Braslau, A.M. Levelut and G. Durand, Surface induced transitions in the nematic phase of 4,n-octyloxybenzoic acid, J. Phys. II (France) 2, 1159-1194 (1992)

[16] P. Simova and M. Petrov, On the electrical conductivity in the nematic phase of 4-n-alkyloxybenzoic acids HOBA, OOBA, and NOBA, Phys. Stat. Sol. A 80. K153-K156, 1983; M. Petrov and P. Simova, Depolarised Rayleigh scattering and textures in the nematic phase of some 4,n-alkyloxybenzoic acids, J. Phys. D: Appl. Phys. 18. 239-246, 1985.

[17] L. Frunza, S. Frunza, A. Sparavigna, M. Petrov and S.I. Torgova, Dielectric and DSC investigations of 4-n-substituted benzoic and cyclohexane carboxylic acids .1. Textural changes in homologous 4-n-alkoxybenzoic acids, Molecular Crystals and Liquid Crystals Science and Technology, Section C - Molecular Materials 6(3), p.215-223, 1996.

[18] A. De Vries, Evidence for the existence of more than one type of nematic phase, Mol. Cryst. Liq. Cryst. 10, 31-35, 1970.

[19] P.J. Martin and D.W. Bruce, Hydrogen-bonded oxadiazole mesogens, Liq. Cryst. 34, 767-774, 2007.

[20] M. Parra, S. Hernández and J. Alderete, Metallomesogens derived from chelating Schiff-bases containing 1,3,4-oxadiazole and 1,3,4-thiadiazole: synthesis, characterization and study of mesomorphic properties, J. Chil. Chem. Soc. 48, 57-60, 2003.

[21] C. Adachi, T. Tsutsui and S. Saito, Blue light-emitting organic electroluminescent devices, Appl. Phys. Lett. 56, 799-781, 1990.

[22] H. Mochizuki, T. Hasui, M. Kawamoto, T. Shiono, T. Ikeda, C. Adachi, Y. Taniguchi and Y. Shirota, Novel liquid-crystalline and amorphous materials containing oxadiazole and amine moieties for electroluminescent devices, Chem. Commun., 1923-1924, 2000 (see references therein)

[23] A.P. Kulkarni, C.J. Tonzola, A. Babel and S.A. Jenekhe, Electron transport materials for Organic Light-Emitting Diodes, Chem. Mater. 16, 4556-4573, 2004.

[24] Y-D. Zhang, K.G. Jespersen, M. Kempe, J.A. Kornfield, S. Barlow, B. Kippelen and S.R. Marder, Columnar discotic liquidcrystalline oxadiazoles as electron-transport materials, Langmuir 19, 6534-6536, 2003.

[25] D.D. Prabhu, N.S. Kumar, A.P. Sivadas, S. Varghese and S.J. Das, Trigonal 1,3,4-oxadiazole-based blue emitting liquid crystals and gels, Phys. Chem. B. 116(43), 13071-80 (2012)

[26] Rong Fan, D. Culjkovic, P. Piromreun, M.J. Turon, J.E. Langseth, G.G. Milliaras, Gu Shihai, L. Sukhomlinova and R.J. Twieg, Light-emitting diodes based on a liquid-crystalline oxadiazole derivative, Proc. SPIE, Organic Light-Emitting Materials and Devices III, Zakya H. Kafafi Ed. 3797, 170-177, 1999. 
[27] M.K. Leung, W-H. Yang, C-N. Chuang, J-H. Lee, C-F. Lin, M$\mathrm{K}$. Wei and Y-H. Liu, 1,3,4-oxadiazole containing silanes as novel hosts for blue phosphorescent Organic Light Emitting Diodes, Org. Lett., 14 (19), 4986-4989, 2012.

[28] V. Sivasubramaniam, F. Brodkorb, S. Hanning, H.P. Loebl, V. van Elsbergen, H. Boerner, U. Scherf, M. Kreyenschmidt, Fluorine cleavage of the light blue heteroleptic triplet emitter FIrpic, Journal of Fluorine Chemistry 130( 7), 640-649, 2009

[29] V. Görtz and J.W. Goodby, Enantioselective segregation in achiral nematic liquid crystals, Chem Commun. 3262-3264, 2005

[30] S-W. Choi, S. Kang, Y. Takanishi, K. Ishikawa, J. Watanabe and $H$. Takezoe, Intrinsic chiral domains enantioselectively segregated from twisted nematic cells of bent core mesogens, Chirality 19, 250-254, 2007.

[31] V.M.L. Braga, S.J. Melo, R.M. Srivastava and E.P. da S. Falcão, Synthesis of new 1,2,4-oxadiazoles carrying (1S)-t-
butyloxycarbonyl-1-amino-(2S)-methyl-1-butyl and (1S)-tbutyloxycarbonyl-1-amino-1-ethyl groups at C-5, J. Braz. Chem. Soc. 15, 603-607, 2004.

[32] M.P. Hutt, E.F. Elslager and L.M. Werbel, 2-Phenyl-5(trichloromethyl)-1,3,4-oxadiazoles, A new class of antimalarial substances, Journal of Heterocyclic Chemistry 7(3), 511-518, 1970

[33] B. Silvestrini and C. Pozzatti, Pharmacological properties of 3phenyl-5 $\beta$ diethylaminoethyl-1,2,4-oxadiazole, $\mathrm{Br} \mathrm{J}$ Pharmacol Chemother. 16(3), 209-217, 1961 .

[34] P. Singh, P.K. Sharma, J.K. Sharma, A. Upadhyay and N. Kumar, Synthesis and evaluation of substituted diphenyl-1,3,4oxadiazole derivatives for central nervous system depressant activity, Organic and Medicinal Chemistry Letters 2(1), Article 8, 10 Pages, 2012. 